\title{
A Massive Degenerative Leiomyoma Mimicking an Ovarian Tumor: A Diagnostic Dilemma
}

\author{
J. Karthiga Prabhu, MD (OBG), Sunita Samal, MD, Shanmugapriya Chandrasekar, MD, \\ Divya Subramani, MD, and Shanmugapriya Rajamanickam, MD
}

\begin{abstract}
Background: Large leiomyomas can outgrow their blood supplies leading to cascades of inflammatory reactions and ischemic changes resulting in various kinds of degeneration. Massive cystic degeneration might mimic an ovarian cyst, posing a diagnostic dilemma. This article presents a case of a woman with marked cystic degeneration of a uterine leiomyoma mimicking an ovarian neoplasm.

Case: A 42-year-old woman presented to the gynecology outpatient clinic of SRM Medical College Hospital and Research Centre, SRM Nagar, Chengalpattu District, Tamil Nadu, India. She had a huge abdominopelvic mass. Clinically and radiologically, the mass was suspected to be an ovarian neoplasm. This patient's tumor markers were normal. Intraoperatively, a huge mass filling the entire abdominal cavity was seen. The mass was decompressed and $4.5 \mathrm{~L}$ of serous fluid were drained. It was attached to the posterolateral wall of the uterus and left ovary. She underwent a total abdominal hysterectomy, a bilateral salpingo-oophorectomy, and an infracolic omentectomy

Results: Histopathology confirmed a final diagnosis of a degenerated leiomyoma. The patient's postoperative course was uneventful and she was discharged on her eighth postoperative day.

Conclusions: When a patient has a huge pelvic mass, cystic degeneration of this fibroid mass should be retained as a differential diagnosis prior to surgical intervention. ( $\mathrm{J}$ GYNECOL SURG 37:67)
\end{abstract}

Keywords: gynecology, leiomyoma, cystic degeneration, hyaline degeneration, ovarian tumor

\section{Introduction}

$\mathbf{L}$ EIOMYOMA OF THE UTERUS is the most-common benign $\boldsymbol{d}$ tumor arising from uterine smooth muscle. The sizes of leiomyomas vary from being microscopic to being giant; giant myomas are exceedingly rare. As leiomyomas enlarge, they outgrow their blood supplies, which can lead to cascades of inflammatory reactions and ischemic changes that result in various kinds of degeneration, such as hyaline, cystic, myxoid, or red degeneration. Hyaline cystic degeneration is relatively rare and has been observed in $\sim 4 \%$ of leiomyomas. ${ }^{1}$ Massive cystic degeneration can mimic ovarian cysts, posing diagnostic dilemmas. This article presents a case of a woman with marked cystic degeneration of an exophytic uterine leiomyoma mimicking an ovarian neoplasm.

\section{Case}

A 42-year-old woman presented to the gynecology outpatient clinic of the SRM Medical College Hospital and
Research Centre, in SRM Nagar, Kattankulathur, Chengalpattu District, Tamil Nadu, India. She had abdominal distension for 1 week prior with occasional abdominal pain. She also experienced loss of appetite for 2 months. She was a nulligravida who had been evaluated for primary infertility 10 years prior. Her menstrual cycles were regular with no dysmenorrhea. There was no other significant medical history. Her general condition was fair with mild pallor with no evidence of any lymphadenopathy. On clinical examination a huge nontender abdominopelvic mass soft to cystic in consistency was palpated extending up to the xiphi sternum with restricted mobility. On bimanual examination it was difficult to differentiate the mass from the uterus. Clinically, the mass was suspected to be an ovarian mass.

Ultrasonography (USG) showed a large $(20 \times 16 \mathrm{~cm})$ cystic mass, with multiple thin septations, internal echoes, and solid components, arising from the pelvis and occupying the entire abdominal cavity (Figs 1 and 2). The uterus was normal with a thin endometrium. The right ovary was normal, but the left

Department of Obstetrics and Gynaecology, SRM Medical College Hospital and Research Centre, SRM Institute of Science and Technology, SRM Nagar, Kattankulathur, Chengalpattu District, Tamil Nadu, India. 


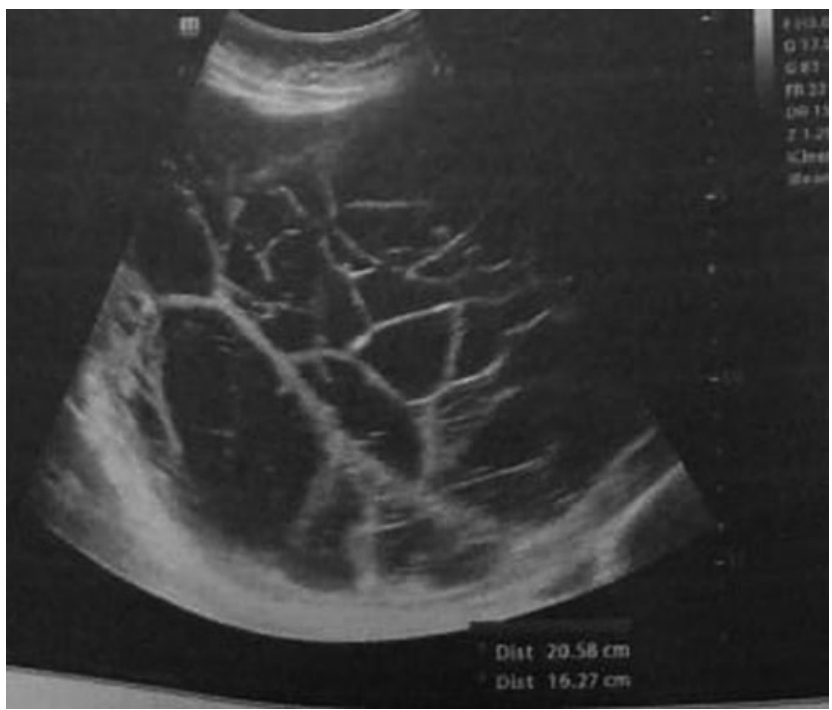

FIG. 1. Ultrasound image showing cystic tumor with multiple septations and internal echoes.

ovary could not be visualized. Thus, a provisional diagnosis of a left ovarian tumor was made. Tumor-marker levels, such as cancer antigen-125-28.96 U/mL and carcinoembryonic antigen-1.610 ng/mL, were normal.

Subsequently, she underwent a laparotomy that revealed the large ovarian mass extending from the pelvis up to the lower border of her rib cage and occupying the entire abdomen. Due to the difficulty involved in removing this huge mass from the abdominal cavity, the mass was decompressed by making a small incision in it. A suction cannula was inserted, and $4.5 \mathrm{~L}$ of serous fluid were drained, with precautions taken to avoid any spillage.

The patient's uterus was normal with normal right adnexa. The huge mass was attached on the left side to the posterolateral wall of the uterus along with the left ovary (Figs. 3 and 4). The consistency of this mass was variegated and predominantly cystic. There was minimal free fluid in the pelvis. Subsequently, the patient underwent a total ab-

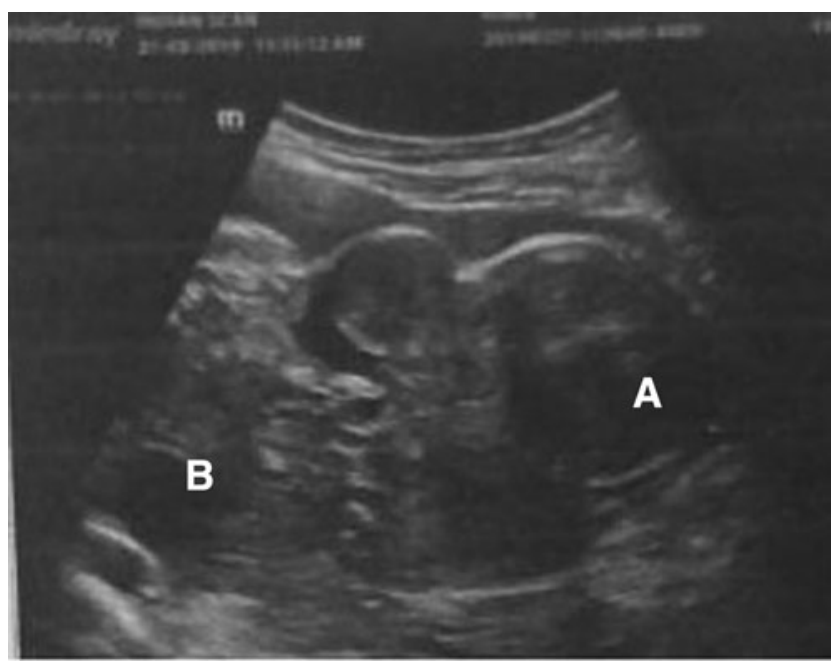

FIG. 2. Ultrasound image showing a part of the tumor with a solid component. A, fibroid; $\mathbf{B}$, mass.

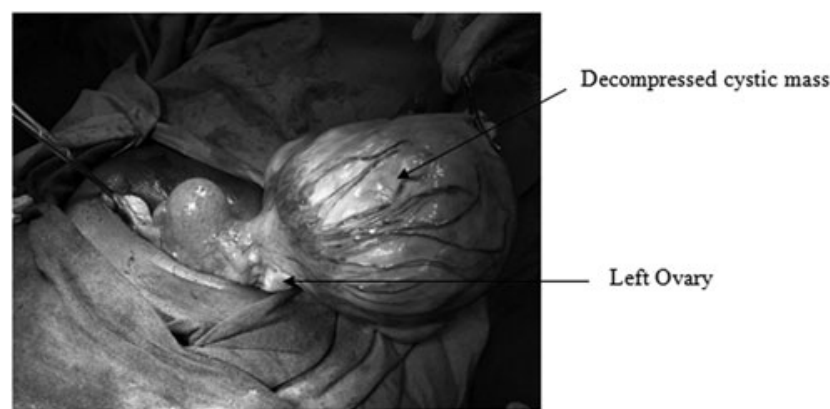

FIG. 3. Decompressed cystic mass with pedunculated attachment to the uterus and left ovary.

dominal hysterectomy, a bilateral salpingo-oophorectomy, and an infracolic omentectomy. The pelvic and para-aortic nodes were not palpable. Peritoneal fluid was sent for cytological examination to rule out malignancy.

\section{Results}

This patient's postoperative course was uneventful, and she was discharged on her eighth postoperative day. Histopathological examination showed that the mass was a subserous leiomyoma with hyaline and myxoid degeneration.

\section{Discussion}

As leiomyomas enlarge, they can outgrow their blood supplies, inducing a series of inflammatory responses and ischemic changes that result in various kinds of degeneration, including hyaline, cystic, myxoid, or red degeneration; and dystrophic calcification. Degenerative or secondary changes are detectable in $\sim 65 \%$ of uterine leiomyomas as follows: hyaline degeneration (63\%); myxoid changes (19\%); calcification (8\%); cystic changes (4\%); fatty degeneration $(3 \%)$; and red degeneration (3\%). ${ }^{1}$ Cystic degeneration is mainly a pseudocyst derived from liquefaction of hyaline changes (in the late stages). Pedunculation of a subserosal fibroid can compromise the blood supply of the fibroid and play a role in the pathogenesis of cystic degeneration. ${ }^{2,3}$

Typical cases of leiomyoma are easy to identify radiologically. However, uterine leiomyomas altered by degenerative changes, can be misdiagnosed. On USG, leiomyomas are

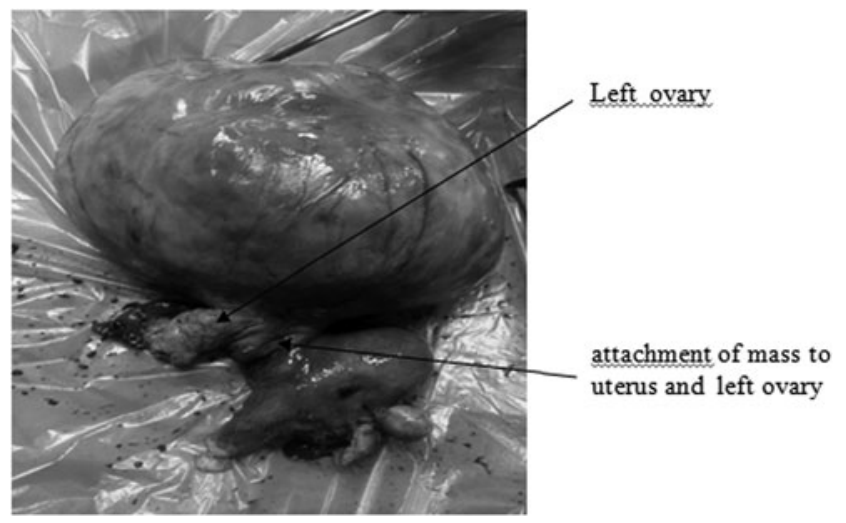

FIG. 4. Hysterectomy specimen: Cystic mass with attachments to both the uterus and the ovary. 
characterized by homogeneous or heterogeneous hypoechoic masses, but, with cystic degeneration, a fibroid can have a variegated appearance. The fibroid can mimic an ovarian cyst, an endometrioma, or an abscess. ${ }^{4,5}$ There are few case reports of degenerative fibroids mimicking ovarian carcinomas. Clinical features and investigations have mimicked ovarian tumors and the diagnoses were made intraoperatively. So far, fewer than 20 cases of degenerated leiomyomas mimicking ovarian tumors have been reported in the literature.

Anyanvu et al. reported 2 case series of cystic degeneration in pregnant and nonpregnant women. ${ }^{9}$ In 1 of these cases, there was a combination of three kinds of degeneration (hyaline, cystic, and myxoid), which is extremely rare; 8.2 L of fluid were drained from that fibroid. ${ }^{9}$

Bhardwaj et al. reported a case of massive cystic degeneration of a fibroid that was aspirated laparoscopically; the surgeons then proceeded with a hysterectomy. ${ }^{10}$ Enakpene et al. reported the largest leiomyoma with cystic degeneration (containing $11 \mathrm{~L}$ of serous fluid) in a nulliparous woman whose case was managed with fertility-preserving surgery. ${ }^{11}$

The first differential diagnosis of a large cystic mass with irregular septae and solid components filling the pelvic cavity is ovarian malignancy. This can be ruled out by visualizing normal ovaries or continuity of the mass with the uterus on imaging. Absence of ascites and elevated tumor markers may favor a diagnosis of a leiomyoma. The ovarian vascular pedicle sign noted on computed tomography (CT) is another way to confirm the ovarian origin of a pelvic mass and for differentiating the mass from a subserosal uterine fibroid. ${ }^{12}$

Maizlin et al. reported a case in which the sonographic features supported the diagnosis of an ovarian tumor but magnetic resonance imaging (MRI) aided the preoperative diagnosis of cystic degenerations of fibroids. ${ }^{13}$ In the current patient's left ovary also could not be visualized on USG. Thus, the role of MRI in choosing the preoperative diagnosis cannot be overemphasized and MRI should be performed in all cases when there are huge cystic masses and adnexae are not visualized. Yorita et al., in a review article, noted that, among 17 cases mimicking ovarian tumors, most $(70 \%)$ were actually subserosal pedunculated masses and 6 cases had multilocular cystic morphology. All 12 ovarian tumorlike uterine leiomyomas could not be clinically diagnosed using imaging modalities, such as ultrasound, CT and/or MRI, partly because of their failure to show the stalk of the pedunculated fibroids. ${ }^{14}$

This case was presented here because of its rarity. Even intraoperatively, the mass was suspected to be a ovarian mass as it was attached to one ovary and to the uterus. Only histopathology testing confirmed the final diagnosis of a degenerated leiomyoma.

\section{Conclusions}

Extensive cystic degeneration in leiomyomas can result in unusual presentations and diagnostic dilemmas. Cystic degeneration of a fibroid should be retained as a differential diagnosis prior to surgical interventions for such type of huge pelvic masses.

\section{Author Disclosure Statement}

No financial conflicts of interest exist.

\section{Funding Information}

No funding was received for this work.

\section{References}

1. Rosai J. Rosai and Ackerman's Surgical Pathology: Female Reproductive System: Vulva, Vagina, Uterus, Fallopian tubes, Ovary, Placenta. 10th ed. London: Mosby, 2011:1510.

2. Hacivelioglu S, Erkanli S. A large pedunculated leiomyoma with two-sided cystic degenerations mimicking a bilateral ovarian malignancy: A case report. Eur J Gynaecol Oncol 2014;35:192.

3. Low SC, Chong CL. A case of cystic leiomyoma mimicking an ovarian malignancy. Ann Acad Med Singapore 2004;33:371.

4. Reddy NM, Jain K, Gerscovich EO. A degenerating cystic uterine fibroid mimicking an endometrioma on sonography. J Ultrasound Med 2003;22:973.

5. Salvo DN. Sonographic imaging of maternal complications of pregnancy. J Ultrasound Med 2003;22:69.

6. Gajewska M, Kosinska-Kaczynska K, Marczewska J, Kaminski P. Huge uterine leiomyoma with degenerative changes mimicking ovarian carcinoma-a case report [in Polish]. Ginekol Pol 2013;84:147.

7. Aydin C, Eris S, Yalcin Y, Sen SH. A giant cystic leiomyoma mimicking an ovarian malignancy. Int J Surg Case Rep 2013;4:1010.

8. Kaushik C, Prasad A, Singh Y, et al. Case series: Cystic degeneration in uterine leiomyomas. Indian J Radiol Imaging 2008;18:69.

9. Anyanwu M, Gassama K, Kandeh M. Diagnostic dilemma of hyaline cystic degeneration of uterine fibroids. Obstet Gynecol Int J 2019;10:202.

10. Bhardwaj P, Singh N, Batra S, KumarDas T. Massive cystic degeneration in uterine fibroid: Laparoscopic management. Curr Med Res Pract 2016;6:245.

11. Enakpene CA, Mechineni KV, Pardo C, Rickett-Holcolm L, Bowers C Jr, Muneyyirci-Delale O. Large cystic degenerated leiomyoma: A case report and review of similar unusual presentations and diagnostic dilemmas in gynecology. J Gynecol Surg 2011;27:299.

12. Lee JH, Jeong YK, Park JK, Hwang JC. "Ovarian vascular pedicle sign" revealing organ of origin of a pelvic mass lesion on helical CT. Am J Roentgenol 2003;181:131.

13. Maizlin ZV, Vos PM, Cooperberg PL. Is it a fibroid? Are you sure? Embolization for symptomatic uterine fibroids (Cochrane Review). Sonography with MRI assistance. Ultrasound Q 2007;23:55.

14. Yorita K, Tanaka Y, Hirano K, et al. A subserosal, pedunculated, multilocular uterine leiomyoma with ovarian tumor-like morphology and histological architecture of adenomatoid tumors: A case report and review of the literature. J Med Case Rep 2016;10:352.

Address correspondence to: J. Karthiga Prabhu, $M D(O B G)$ Plot-45, Vallalar Street Chelli Nagar Selaiyur, Chennai-600073 Tamil Nadu India

E-mail: j.karthigaprabhu@gmail.com 